\title{
Mechanics of thin-film transistors and solar cells on flexible substrates
}

\author{
Helena Gleskova*, I-Chun Cheng, Sigurd Wagner, and James C. Sturm \\ Department of Electrical Engineering and Princeton Institute for the Science \\ and Technology of Materials, Princeton University, Princeton, NJ 08544, USA
}

\section{Zhigang Suo}

Division of Engineering and Applied Sciences, Harvard University, Cambridge, MA 02139, USA

When devices are fabricated on thin foil substrates, any mismatch strain in the device structure makes the work piece curve. Any change of the radius of curvature produces a change in the size of the work piece, and thereby misalignment between individual device layers. To achieve tight tolerances, changes of curvature must be minimized throughout the fabrication process.

Amorphous silicon thin-film transistors and solar cells respond differently to externally applied tensile strain. The elastic deformation of the transistor is correlated with small increase in the electron mobility. When the tensile strain reaches $\sim 0.34 \%$, crack formation starts and causes an abrupt change in the transistor performance. The performance of solar cells, on the other hand, does not change for tensile strain up to $0.7 \%$. At larger strain the short-circuit current, open-circuit voltage, fill factor, and the efficiency gradually decrease.

Key words: amorphous silicon, thin-film transistor, solar cell, flexible electronics

Phone: (609) 258-4626, Fax: (609) 258-3585, E-mail: gleskova@princeton.edu 


\section{Introduction.}

Recent research in thin-film electronics has been focused on the replacement of the traditional rigid glass plate substrate with plastic or metallic foils. Among metallic materials, stainless steel and molybdenum foils have been utilized as substrates in the fabrication of thin-film transistors (Theiss and Wagner, 1996; Wu et al., 1997; Howell et al., 2000; Wu et al., 2002; Park et al., 2003) and solar cells (Yang et al., 2003). A number of plastic materials (organic polymers) also have been tested successfully in a variety of thin-film applications (Constant et al., 1994; Young et al., 1997;Burns et al., 1997; Burrows et al., 1997; Gleskova et al., 1998; Parsons et al., 1998; Lueder et al., 1998; Thomasson et al., 1998; Sandoe, 1998; Carey et al., 2000; Sazonov et al., 2000; Boucinha et al., 2000; Kane et al., 2001; Ichikawa et al., 2001; Hsu et al., 2002a; Brida et al., 2002; Takano et al., 2003; Cheng et al., 2004; Gelinck et al., 2004; Shahrjerdi et al., 2004; Nomura et al., 2004; Monacelli et al., 2004; Choi et al., 2004).

There are three main reasons for the attraction of plastic and metallic foils. Unlike glass, the thickness of these materials can be substantially reduced while maintaining their integrity, leading to thin and lightweight products. At the same time, these thin substrates add new functionality to thin-film electronics, namely the flexing and non-planar shaping. Finally, the foil substrates lend themselves to roll-to-roll fabrication.

One faces several new issues when fabricating devices on thin foils. These are usually not encountered during the fabrication of these devices on thick plates of glass. Firstly, the devices experience variable stresses during the manufacturing process that may lead to substantial change in curvature. This leads to a change in the size of the 
work piece and ultimately to misalignment between different layers of the device. This is important for devices where mask overlay alignment is critical. Therefore, the radius of curvature must be carefully controlled during the fabrication. Secondly, the device application may require intentional bending, stretching, or non-planar shaping after the fabrication. Therefore, one needs to understand the behavior of thin-film devices under strain, and the fracture strain and fracture mechanism of the device layers.

Even though a detailed understanding and comprehensive mechanical theory do not yet exist, a number of experimental results are available and simple mechanical theories have been worked out (Gleskova and Wagner, 1999a; Suo et al., 1999; Gleskova and Wagner, 1999b; Gleskova et al., 2000; Wagner et al., 2000; Gleskova and Wagner, 2001; Hsu et al., 2002b; Wagner et al., 2002; Gleskova et al., 2002; Jones et al., 2002; Hsu et al., 2004; Gleskova et al., 2004; Servati and Nathan, 2005). The purpose of this paper is to summarize the current knowledge of the mechanics of thin-film electronics with a focus on amorphous silicon thin-film technology. In the calculations we emphasize two-layer structures of substrate and film. Such structures are simple enough to be treated analytically, yet they provide a basic understanding of the mechanics of thin-film devices on flexible substrates.

\section{Curvature induced during manufacturing.}

Thin-film devices are built on substrates layer-by-layer, often at elevated temperature. Strain develops in the structure by built-in stresses in the deposited layers (Hooke's law: $\sigma=Y \cdot \varepsilon$, where $\sigma$ is stress, $Y$ Young's modulus, and $\varepsilon$ strain), or, upon cooling down, by the differences in the thermal expansion and humidity coefficients 
between the deposited film and the substrate, or between different films. The mechanics of the film-on-substrate structure depends strongly on the elastic (Young's) moduli and thicknesses of the substrate $Y_{s}, d_{s}$ and the thin film $Y_{f}, d_{f}$.

When $Y_{f} \cdot d_{f}<<Y_{s} \cdot d_{s}$, the substrate dominates and the film complies with it, as a thin-film transistor (TFT) or solar cell do on a plate glass substrate. The stress in the substrate is small, and the film/substrate couple curves only slightly, even when the film is highly stressed. The strain is biaxial, and the structure forms a spherical cap with the radius of curvature given by:

$$
R=\frac{d_{s}}{6 \cdot \frac{Y_{f}^{*}}{Y_{s}^{*}} \cdot \frac{d_{f}}{d_{s}} \cdot \varepsilon} \cdot \frac{\left[1-\frac{Y_{f}^{*}}{Y_{s}^{*}} \cdot\left(\frac{d_{f}}{d_{s}}\right)^{2}\right]^{2}+4 \cdot \frac{Y_{f}^{*}}{Y_{s}^{*}} \cdot \frac{d_{f}}{d_{s}} \cdot\left(1+\frac{d_{f}}{d_{s}}\right)^{2}}{1+\frac{d_{f}}{d_{s}}}
$$

Here $Y_{f}^{*}=\frac{Y_{f}}{1-v_{f}}$ and $Y_{s}^{*}=\frac{Y_{s}}{1-v_{s}}$ are the biaxial strain moduli of the film and substrate, respectively. $v_{f}$ and $v_{s}$ are the corresponding Poisson ratios. $\varepsilon$ is the mismatch strain. The mismatch strain $\varepsilon$ has two dominant components. One is the thermal mismatch strain caused by the difference between the coefficient of thermal expansion of the substrate, $\alpha_{s}$, and that of the film, $\alpha_{f}$. The other is the built-in strain $\varepsilon_{b i}$ in the deposited film. Therefore,

$$
\varepsilon=\left(\alpha_{f}-\alpha_{s}\right) \cdot \Delta T+\varepsilon_{b i}
$$

where $\Delta T$ is the difference between the deposition and the room temperatures. $R$ is negative when the film is under compression and the structure curves with the film being on the convex side. $R$ is positive when the film is under tension and the structure curves with the film being on the concave side. 
For amorphous silicon devices on glass substrates $Y_{f} \cdot d_{f}<<Y_{s} \cdot d_{s}$, which allows to neglect the second fraction in equation (1) and to simplify to the Stoney formula (Freund, 1996; Finot et al., 1997; Cu, 1998):

$$
R=\frac{d_{s}}{6 \cdot \frac{Y_{f}^{*}}{Y_{s}^{*}} \cdot \frac{d_{f}}{d_{s}} \cdot \varepsilon}
$$

In such cases $R$ is very large.

A stiff film and a compliant substrate, for example amorphous silicon device on an organic polymer foil, may have similar products of elastic modulus and thickness, $Y_{f} \cdot d_{f} \approx Y_{s} \cdot d_{s}$. Such equal strength of film and substrate gives rise to complicated mechanical situations. The structure rolls into a cylinder instead of forming a spherical cap. An example is shown in figure 1, for a 500-nm thick layer of silicon nitride deposited on two different plastic substrates by plasma enhanced chemical vapor deposition (PECVD) at $150^{\circ} \mathrm{C}$. The Stoney formula is no longer valid. Using the procedure outlined in (Suo et al., 1999), the radius of curvature $R$ is given by:

$$
\begin{aligned}
& R=\frac{d_{s}}{6 \cdot \frac{Y_{f}^{\prime}}{Y_{s}^{\prime}} \cdot \frac{d_{f}}{d_{s}} \cdot \varepsilon} \cdot\left\{\frac{\left\{\left[1-\frac{Y_{f}^{\prime}}{Y_{s}^{\prime}} \cdot\left(\frac{d_{f}}{d_{s}}\right)^{2}\right]^{2}+4 \cdot \frac{Y_{f}^{\prime}}{Y_{s}^{\prime}} \cdot \frac{d_{f}}{d_{s}} \cdot\left(1+\frac{d_{f}}{d_{s}}\right)^{2}\right\}\left[\left(1-v_{s}^{2}\right)+\left(\frac{y_{f}}{Y_{s}^{\prime}}\right) \cdot\left(1+\frac{Y_{f}^{\prime}}{Y_{s}^{\prime}} \cdot \frac{d_{f}}{d_{s}}\right) \cdot\left[\left(1-v_{s}^{2}\right)\left(1+v_{f}\right)+\frac{Y_{f}^{\prime}}{Y_{s}^{\prime}} \cdot \frac{d_{f}}{d_{s}}\left(1-v_{f}^{2}\right)\left(1+v_{s}\right)\right]\right.}{\left.\left(1-v_{f}^{2}\right)\right]}\right. \\
& \left.+\frac{3 \cdot\left(\frac{Y_{f}^{\prime}}{Y_{s}^{\prime}} \cdot \frac{d_{f}}{d_{s}}\right)^{2}\left(1+\frac{d_{f}}{d_{s}}\right)^{2}\left[\left(1-v_{s}^{2}\right)+\left(1-v_{f}^{2}\right)\right]+2 \cdot \frac{Y_{f}^{\prime}}{Y_{s}^{\prime}} \cdot \frac{d_{f}}{d_{s}} \cdot\left(1-v_{s} v_{f}\right) \cdot\left(1+\frac{Y_{f}^{\prime}}{Y_{s}^{\prime}} \cdot \frac{d_{f}}{d_{s}}\right) \cdot\left(1+\frac{Y_{f}^{\prime}}{Y_{s}^{\prime}} \cdot\left(\frac{d_{f}}{d_{s}}\right)^{3}\right)}{\left(1+\frac{d_{f}}{d_{s}}\right) \cdot\left(1+\frac{Y_{f}^{\prime}}{Y_{s}^{\prime}} \cdot \frac{d_{f}}{d_{s}}\right) \cdot\left[\left(1-v_{s}^{2}\right)\left(1+v_{f}\right)+\frac{Y_{f}^{\prime}}{Y_{s}^{\prime}} \cdot \frac{d_{f}}{d_{s}}\left(1-v_{f}^{2}\right)\left(1+v_{s}\right)\right]}\right\}
\end{aligned}
$$


Here $Y_{f}^{\prime}=\frac{Y_{f}}{1-v_{f}^{2}}$ and $Y_{s}^{\prime}=\frac{Y_{s}}{1-v_{s}^{2}}$ are the plane strain moduli of the film and the substrate, respectively. If the Poisson ratios $v$ of the film and the substrate are identical, equation (4) simplifies into the form published previously (Suo et al., 1999):

$$
R=\frac{d_{s}}{6 \cdot \frac{Y_{f}}{Y_{s}} \cdot \frac{d_{f}}{d_{s}} \cdot \varepsilon} \cdot \frac{\left[1-\frac{Y_{f}}{Y_{s}} \cdot\left(\frac{d_{f}}{d_{s}}\right)^{2}\right]^{2}+4 \frac{Y_{f}}{Y_{s}} \cdot \frac{d_{f}}{d_{s}} \cdot\left(1+\frac{d_{f}}{d_{s}}\right)^{2}}{(1+v) \cdot\left(1+\frac{d_{f}}{d_{s}}\right)}
$$

The mismatch strain $\varepsilon$ is again given by equation (2). When the mechanical properties of the film and the substrate and the radius of curvature $R$ are known, one can easily extract the built-in strain $\varepsilon_{b i}$ in the film (Cheng et al., 2005). Our current focus, however, is on the understanding of how to keep the radius of curvature large, and preferably infinite, since this is an important factor in the device fabrication.

From analysis of equation (5) one can easily conclude that making $Y_{f} \cdot d_{f}<<$ $Y_{s} \cdot d_{s}$ makes $R$ large. This can be achieved by: (1) choosing a thick substrate (not desirable for flexing or "shaping" applications), (2) keeping the device structure very thin (may not be possible), (3) choosing a substrate with large Young's modulus (not possible for plastic substrates), or a combination of these measures. $R$ can also be made large by keeping the mismatch strain $\varepsilon$ close to zero. One can: (1) choose a substrate with a coefficient of thermal expansion (CTE) close to that of the device layers. (This is not possible for plastic substrates whose CTE usually is much larger than that of silicon device films. Stainless steel substrates have relatively low CTE.) (2) minimize $\Delta T$ by lowering the deposition temperature. (In thin-film silicon technology, lower deposition temperature leads to worse electronic properties. Here, the organic electronics, for 
example polymer light emitting diodes and thin-film transistors have an advantage.) (3) compensate the CTE mismatch with built-in strain in the film, such that $\left(\alpha_{f}-\alpha_{s}\right) \cdot \Delta T=\varepsilon_{b i}$ (The built-in strain depends on the deposition conditions and sometimes can be easily adjusted.)

There are two more solutions for dealing with undesirable curving. One is to attach the substrate foil to a rigid carrier for the duration of the fabrication. The rigid carrier suppresses the bending of the structure, and sets the strain in the plane of the film by the thermal strain of the carrier, which is small. This approach, used by a number of research groups, allows a larger selection of plastic materials. However, one needs an adhesive whose glass or decomposition temperature is higher than the highest process temperature. In addition, the adhesive should not outgas in the vacuum and should resist wet and dry processing. Once the fabrication is completed, the adhesive should permit easy separation of the carrier and the work piece. Even though this approach has been demonstrated in the laboratory, it is not an ideal manufacturing solution. The second solution is based on the fact that the patterning of continuous layers into islands relieves the global mismatch strain. However, strain is concentrated around the edges of the islands.

\section{Misalignment caused by curvature.}

Figure 2 shows several thin films typically used in the amorphous silicon thinfilm transistor fabrication (a-Si:H TFT) deposited on $50 \mu \mathrm{m}$ thick Kapton E. As described in the previous section, different thin film materials exhibit different built-in strains resulting in different radii of curvature $R$. It has been shown experimentally that a 
change in curvature affects the alignment between different layers of the device (Cheng et al., 2005). If one starts with a flat substrate, the misalignment increases with decreasing radius of curvature, because a larger strain is developed when the work piece is flattened for mask alignment. The larger the radius of curvature, the less flattening is required and the smaller is the misalignment. In a-Si:H TFTs on Kapton E, the misalignment between the gate and the source/drain electrodes as a function of the deposition power of the gate dielectric easily can reach values of $500 \mathrm{ppm}$. In an optimized structure the misalignment was reduced to $\sim 100 \mathrm{ppm}$ (Cheng et al., 2005). Therefore, the radius of curvature needs to be carefully controlled throughout the fabrication. We are currently developing a mathematical model of this phenomenon with the aim to control it.

\section{Externally applied strain}

All integrated circuits are fabricated flat. The use of the fabricated circuit may require that it is bent (once or repeatedly), stretched, or shaped after the fabrication. Therefore, it is important to understand the electrical and mechanical behavior of the devices under externally applied strain.

a-Si:H TFTs respond to increasing mechanical strain by elastic deformation followed by fracture. The response of the TFT is determined by the magnitude of the applied strain regardless of its origin, for example, bending, stretching or shaping (Suo et al., 1999; Gleskova and Wagner, 2001; Gleskova et al., 2002; Hsu et al., 2004; Gleskova et al., 2004). Experimental findings of the mechanical behavior of the a-Si:H TFTs under externally applied strain are summarized in figure 3. Three different regimes have been 
identified. Under elastic deformation (which is the safe regime) the current-voltage characteristics of the TFT change reversibly (Gleskova and Wagner, 2001; Gleskova et al., 2002; Gleskova et al., 2004; Servati and Nathan, 2005). The electron mobility decreases linearly with compressive strain and increases with tensile strain. The mobility changes correlate with the broadening or steepening of the conduction band tail in a-Si:H channel material. These changes in the electron mobility are relatively small, for example, at the compressive strain of $1 \%$, the mobility is reduced by $25 \%$.

a-Si:H TFTs can be strained more in compression than in tension. No mechanical failure was observed in compression for strains up to $\sim 2 \%$. In tension, the crack formation starts at the strain of $\sim 0.3 \%$ that marks the onset of the transition regime. In the transition regime the TFT fails but the electrical function is restored when the strain is eliminated (Gleskova et al., 2002). When the tensile strain reaches $\sim 0.5 \%$ the cracks become permanent and definitive mechanical failure occurs (Gleskova and Wagner, 1999b). The cracks form perpendicularly to the strain direction. If the source-drain current path and the strain direction are parallel, the cracks interrupt the current path. This is schematically illustrated in figure 4 .

One study suggested that the failure mechanism of the amorphous silicongermanium solar cells is somewhat different from that of TFTs. The results of the bending of triple-junction solar cells on stainless steel substrate (Jones et al., 2002) are summarized in figure 5. No changes in the electrical performance, namely the shortcircuit current $J_{s c}$, open-circuit voltage $V_{o c}$, fill factor $F F$, and efficiency $\eta$, were observed for tensile strain up to $\sim 0.7 \%$ and compressive strain up to $\sim 1.7 \%$. For tensile strains larger than $0.7 \%$, a gradual decrease in $J_{s c}, V_{o c}, F F$, and $\eta$ occurred. However, even at the tensile strain of $2 \%$ (the largest strain applied) the solar cell efficiency was still equal 
to $50 \%$ of the original value. This behavior can be explained by a vertical current path that is parallel to the cracks as illustrated in figure 6 .

\section{Summary.}

The fabrication of thin-film devices on flexible substrates introduces several new fabrication issues that are not encountered during the fabrication of thin-film devices on rigid substrates. Strain that develops in the structure as a result of the built-in stresses in the deposited layers, or differences in the thermal expansion coefficients between the deposited films and the substrate, lead to a change in curvature of the work piece. Any change in the radius of curvature directly affects the misalignment between device levels and produces mask misalignment. To minimize the misalignment, one needs to carefully control the curvature of the work piece throughout the fabrication process.

Amorphous silicon solar cells and thin-film transistors respond differently to an externally applied strain. This difference is more apparent under tension. TFTs start to fail at a strain of $\sim 0.3 \%$ and the failure is abrupt. Solar cells do not exhibit any change in the electrical performance for strains up to $\sim 0.7 \%$. If the tensile strain is further increased, the short-circuit current $J_{s c}$, open-circuit voltage $V_{o c}$, fill factor $F F$, and the efficiency $\eta$ gradually decrease. The horizontal current flow in a TFT in contrast to the predominantly vertical current flow in a solar cell accounts for the difference.

\section{Acknowledgement.}


The authors gratefully acknowledge support from the New Jersey Commission on Science and Technology.

\section{Nomenclature.}

$d \quad$ layer thickness ( $\mathrm{m}$ ); subscript $s$ denotes substrate, $f$ denotes film

FF solar cell fill factor

$J_{s c} \quad$ short circuit current (A)

$R \quad$ radius of curvature (m)

$T \quad$ temperature $(\mathrm{K})$

$V_{o c} \quad$ open-circuit voltage (V)

Y Young's modulus (Pa); subscript $s$ denotes substrate, $f$ denotes film

$Y^{*}=\frac{Y}{1-v} \quad$ biaxial stain modulus $(\mathrm{Pa})$; subscript $s$ denotes substrate, $f$ denotes film

$Y^{\prime}=\frac{Y}{1-v^{2}} \quad$ plain strain modulus $(\mathrm{Pa})$; subscript $s$ denotes substrate, $f$ denotes film

$\alpha$

coefficient of thermal expansion $\left(\mathrm{K}^{-1}\right)$; subscript $s$ denotes substrate, $f$ denotes film

$\varepsilon \quad$ mechanical strain

$\varepsilon_{b i} \quad$ built-in strain in the film

$v \quad$ Poisson ratio; subscript $s$ denotes substrate, $f$ denotes film

$\eta \quad$ solar cell efficiency

$\sigma \quad$ mechanical stress $(\mathrm{Pa})$ 


\section{References:}

Boucinha, M., Brogueira, P., Chu, V., Conde, J.P., 2000. Amorphous silicon air-gap resonators on large-area substrates, Appl. Phys. Lett. 77, 907-909.

Brida, D., Fortunato, E., Aguas, H., Silva, V., Marques, A., Pereira, L., Ferreira, I., Martins, R., 2002. New insights on large area flexible position sensitive detectors. J. Non-cryst. Solids 299, 1272-1276.

Burns, S.G., Shanks, H., Constant, A., Gruber, C., Schmidt, D., Landin, A., Olympie, F., 1997. Design and fabrication of high current switching TFTs on flexible polyimide substrates, In: Kuo, Y. (Ed.), The Electrochemical Society Proceedings, Vol. 96-23, pp. 382-390.

Burrows, P.E., Gu, G., Bulović, V., Shen, Z., Forrest, S.R., Thompson, M.E., 1997, Achieving full-color organic light-emitting devices for lightweight, flat-panel displays. IEEE Trans. Electron Dev. 44, 1188-1203.

Carey, P.G., Smith P.M., Theiss, S.D., Wickboldt, P., 2000. Polysilicon thin-film transistors fabricated on low temperature plastic substrates. J. Vac. Sci. Technol. A17, 1946-1949.

Cheng, I.-C., Wagner, S., 2004. Monolithically integrated p- and n-channel thin film transistors of nanocrystalline silicon on plastic substrates (2004), In: Ganguly, G., Kondo, M., Schiff, E.A., Carius, R., Biswas, R. (Eds.), Amorphous and Nanocrystalline Silicon Science and Technology - 2004, Mat. Res. Soc. Symp. Proc. 808, pp. A4.6, 703-708.

Cheng, I.C., Kattamis, A., Long, K., Sturm, J.C., Wagner, S., 2005. Stress control for overlay registration in a-Si:H TFTs on flexible organic polymer foil substrates. J. SID, to be published. 
Choi, H.Y., Kim, S.H., Jang, J., 2004. Self-organized organic thin-film transistors on plastic. Adv. Mater. 16, 732-736.

Constant, A., Burns, S.G., Shanks, H., Gruber, C., Landin, A., Schmidt, D., Thielen, C., Olympie, F., Schumacher, T., Cobbs, J., 1994. Development of thin-film transistor based circuits on flexible polyimide substrates. In: Kuo, Y. (Ed.), The Electrochemical Society Proceedings, Vol. 94-35, pp. 392-400.

$\mathrm{Cu}$, S.N.G., 1998. Elastic bending of semiconductor wafer revisited and comments on Stoney’s equation. J. Electrochem. Soc. 145, 3621-3627.

Finot, M., Blech, I.A., Suresh, S., Fujimoto, H., 1997. Large deformation and geometric instability of substrates with thin-film deposits. J. Appl. Phys. 81, 3457-3464.

Freund, L.B., 1996. Some elementary connections between curvature and mismatch strain in compositionally graded thin films. J. Mech. Phys. Solids 44, 723-736.

Gelinck, G.H., Huitema, H.E.A., van Veenendal, E., Cantatore, E., Schrijnemakers, L., van der Putten, J.B.P.H., Geuns, T.C.T., Beenhakkers, M., Giesbers, J.B., Huisman, B.-H., Meijer, E.J., Benito E.M., Touwslager, F.J., Marsman, A.W., van Rens, B.J.E., De Leeuw, D.M., 2004. Flexible active-matrix displays and shift registers based on solution-processed organic transistors, Nature Materials 3, 106-110.

Gleskova, H., Wagner, S., Suo, Z., 1998. a-Si:H TFTs made on polyimide foil by PECVD at $150^{\circ}$ C. In: Parsons, G.N., Tsai, C.C., Fahlen, T.S., Seager, C.H. (Eds.), Flat Panel Display Materials - 1998, Mat. Res. Soc. Symp. Proc. 508, pp. 73-78.

Gleskova, H., Wagner, S., 1999a. Amorphous silicon thin film transistors on compliant polyimide foil substrates. IEEE Electron Dev. Lett. 20, 473-475.

Gleskova, H., Wagner, S., 1999b. Failure resistance of amorphous silicon transistors under extreme in-plane strain. Appl. Phys. Lett. 75, 3011-3013. 
Gleskova, H., Wagner, S., Suo, S., 2000. a-Si:H thin film transistors after very high strain. J. Non-cryst. Solids 266-269, 1320-1324.

Gleskova, H., Wagner, S., 2001. Electron mobility in amorphous silicon thin film transistors under compressive strain. Appl. Phys. Lett. 79, 3347-3349.

Gleskova, H., Wagner, S., Soboyejo, W., Suo, Z., 2002. Electrical response of amorphous silicon thin-film transistors under mechanical strain. J. Appl. Phys. 92, 6224-6229.

Gleskova, H., Hsu, P.I., Xi, Z., Sturm, J.C., Suo, Z., Wagner, S., 2004. Field-effect mobility of amorphous silicon thin-film transistors under strain. J. Non-cryst. Solids $338-340,732-735$.

Howell, R.S., Stewart, M., Karnik, S.V., Saha, S.K., Hatalis M.K., 2000. Poly-Si thinfilm transistors on steel substrates, IEEE Electron Dev. Lett. 21, 70-72.

Hsu, P.I., Bhattacharya, R., Gleskova, H., Huang, M., Xi, Z., Suo, Z., Wagner, S., Sturm, J.C., 2002a. Thin-film Transistor Circuits on Large-Area Spherical Surfaces. Appl. Phys. Lett. 81, 1723-1725.

Hsu, P.I., Gleskova, H., Huang, M., Suo, Z., Wagner, S., Sturm J.C., 2002b. Amorphous Si TFTs on plastically deformed spherical domes. J. Non-cryst. Solids 299-302, 13551359.

Hsu, P.I., Huang, M., Gleskova, H., Xi, Z., Suo, Z., Wagner, S., Sturm J.C., 2004. Effect of mechanical strain on TFTs on spherical domes. IEEE Trans. Electron Dev. 51, $371-377$.

Ichikawa, Y., Yoshida, Y., Hama, T., Sakai, H., Harashima, K., 2001. Production technology for amorphous silicon based flexible solar cells. Solar Energy Mat. and Solar Cells 66, 107-115. 
Jones, R., Johnson, T., Jordan, W., Wagner, S., Yang, J., Guha, S., 2002. Effects of mechanical strain on the performance of amorphous silicon triple-junction solar cells. Proc. of the $29^{\text {th }}$ IEEE Photovoltaic Specialists Conference, New Orleans, pp. 12141217.

Kane, M.G., Hill, I.G., Campi, J., Hammond, M.S., Greening, B., Sheraw, C.D., Nichols, J.A., Gundlach, D.J., Huang, J.R., Kuo, C.C., Jia, L., Jackson, T.N., West J.L., Francl, J., 2001. AMLCDs using organic thin-film transistors on polyester substrates. In: Morreale, J. (Ed.), Society for Information Display, Digest of Technical Papers, Vol. 32, San Jose, CA, pp. 57-59.

Lueder, E., Muecke, M. and Polach, S., 1998. Reflective FLCDs and PECVD-generated a-Si-TFTs with plastic substrates. In: Conf. Proc., 18th International Display Research Conference Asia Display '98, Seoul, Korea, Society for Information Display, pp. 173-177.

Monacelli, B., Kotter, D., Boreman, G.D., 2004. Design of a thin-film infrared barcode on a flexible substrate. Internat. J. Infrared and Millimeter Waves 25, 317-325.

Nomura, K., Ohta, H., Takagi, A., Kamyia, T., Hirano, M., Hosono, H., 2004. Room temperature fabrication of transparent flexible thin-film transistors using amorphous oxide semiconductors. Nature 432, 488-492.

Park, J.H., Kim, D.Y., Ko, J.K., Chakrabarty, K., Yi, J., 2003. High temperature crystallized poly-Si on Mo substrates for TFT application. Thin Solid Films 427, 303-308.

Parsons, G. N., Yang, C. S., Arthur, C. B., Klein, T. M. and Smith, L., 1998. Reaction processes for low temperature plasma enhanced deposition of hydrogenated amorphous silicon thin film transistors on transparent plastic substrates. In: Parsons, 
G.N., Tsai, C.C., Fahlen, T.S., Seager, C.H. (Eds.), Flat Panel Display Materials 1998, Mat. Res. Soc. Symp. Proc. 508, pp. 19-24.

Sandoe, J.N., 1998. AMLCD on plastic substrates. In: Morreale, J. (Ed.), Society for Information Display, Digest of Technical Papers, Vol. 29, Santa Ana, CA, pp. 293296.

Sazonov A., Nathan A., 2000. $120^{\circ} \mathrm{C}$ fabrication technology for a-Si:H thin-film transistors on flexible polyimide substrates. J. Vac. Sci. Technol. A18, 780-782.

Servati, P., Nathan, A., 2005. Orientation-dependent strain tolerance of amorphous silicon transistors and pixel circuits for elastic organic light-emitting diode displays. Appl. Phys. Lett. 68, 033504.

Shahrjerdi, D., Hekmatshoar, B., Mohajerzadeh, S.S., Khakifirooz, A., Robertson, M., 2004. High mobility poly-Ge thin-film transistors fabricated on flexible plastic substrates at temperatures below $130^{\circ} \mathrm{C}$. J. Electronic Materials 33, 353-357.

Suo, Z., Ma, E.Y., Gleskova, H., Wagner, S., 1999. Mechanics of rollable and foldable film-on-foil electronics. Appl. Phys. Lett. 74, 1177-1179.

Takano, A., Tanda, M., Shimozawa, M., Wada, T., Kamoshita, T., 2003. Excitation frequency effects on stabilized efficiency of large-area amorphous silicon solar cells using flexible plastic substrate. Jap. J. Appl. Phys., Part 2, 42, L1312-L1314.

Theiss, S. D., Wagner, S., 1996. Amorphous silicon thin-film transistors on steel foil substrates. IEEE Electron Dev. Lett. 17, 578-580.

Thomasson, D.B., Bonse, M., Huang, J.R., Wronski, C.R., Jackson, T.N., 1998. Tri-layer a-Si:H integrated circuits on polymeric substrates. In: Conf. Record 1998 International Electron Devices Meeting, IEEE, pp. 253-256. 
Wagner S., Gleskova H., Sturm, J.C., Suo, Z., 2000. Novel processing technology for macroelectronics, in: Street, R.A. (Ed.), Technology and applications of amorphous silicon, Springer, Berlin, pp. 222-251.

Wagner, S., Gleskova, H., Cheng, I.C., Wu, M., 2002. Thin film transistors and flexible electronics. In: Bergmann, R.B. (Ed.), Growth, characterization and electronic applications of Si-based thin films, Research Singpost, Kerala, India, pp. 1-14.

Wagner, S., Gleskova, H., Cheng, I.-C., Sturm, J.C., Suo, Z., 2005. Mechanics of TFT technology on flexible substrates, In: Crawford, G.P. (Ed.), Flexible Flat Panel Displays, John Wiley \& Sons, Chichester, England, 263-283.

Wu, C.C., Theiss, S.D., Gu, G., Lu, M.H., Sturm, J.C., Wagner, S., Forrest, S.R., 1997. Integration of organic LEDs and amorphous Si TFTs onto flexible and lightweight metal foil substrates. IEEE Electron Dev. Lett. 18, 609-612.

Wu, M., Bo, X.Z., Sturm, J.C., Wagner, S., 2002. Complementary metal-oxidesemiconductor thin-film transistor circuits from a high-temperature polycrystalline silicon process on steel foil substrates. IEEE Trans. Electron Devices 49, 1993 2000.

Yang, Y., Banerjee, A., Guha, S., 2003. Amorphous silicon based photovoltaics - from earth to the "final frontier". Solar Energy Mat. and Solar Cells 78, 597-612.

Young, N.D., Bunn, R.M., Wilks, R.W., McCulloch, D.J., Deane, S.C., Edwards, M.J., Harkin, G., Pearson, A.D., 1997. Thin-film-transistor- and diode-addressed AMLCDs on polymer substrates, J. SID 5, 275-281. 


\section{Figure captions:}

Figure 1. PECVD silicon nitride film deposited on two different plastic substrates. The substrate thickness and the coefficient of the thermal expansion are shown for each substrate. The film is deposited on the top and the structures roll into cylinder.

Figure 2. Curvature induced by mismatch strain in films of $\mathrm{Cr}$, a-Si:H, and $\mathrm{SiN}_{\mathrm{x}}$ deposited over a range of RF power, all on $50-\mu$ m thick Kapton ${ }^{\circledR} 200 \mathrm{E}$ polyimide substrates. All films are facing to the left. The 300-nm to 500-nm thick $\mathrm{SiN}_{\mathrm{x}}$ and the 250-nm thick a-Si:H films were deposited by PECVD at $150^{\circ} \mathrm{C}$, and the $80-\mathrm{nm}$ thick $\mathrm{Cr}$ was deposited by thermal evaporation without control of substrate temperature. The builtin stress of $\mathrm{Cr}$ is tensile and that of a-Si:H is compressive. The built-in stress in $\mathrm{SiN}_{\mathrm{x}}$ changes with the deposition power. [47]

Figure 3. Summary of the response of a-Si:H TFT on Kapton E to mechanical strain. [47]

Figure 4. Crack formation in a TFT when the strain and the source-drain current path are paralel. The arrow depicts the current path.

Figure 5. Summary of the response of a triple-juction amorphous silicon-germanium solar cell on stainless steel to mechanical strain.

Figure 6. Crack formation in a solar cell with respect to the current path. 


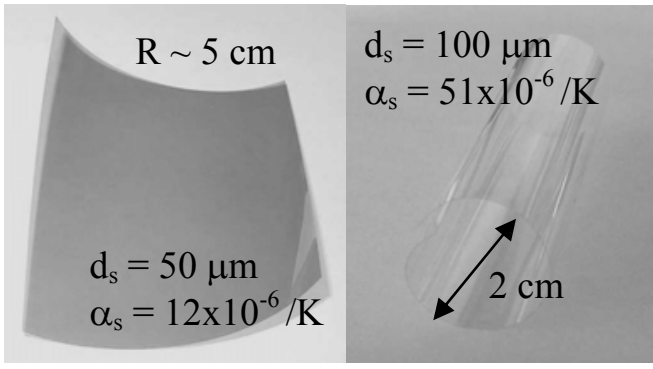

Figure 1.

Gleskova et al. 


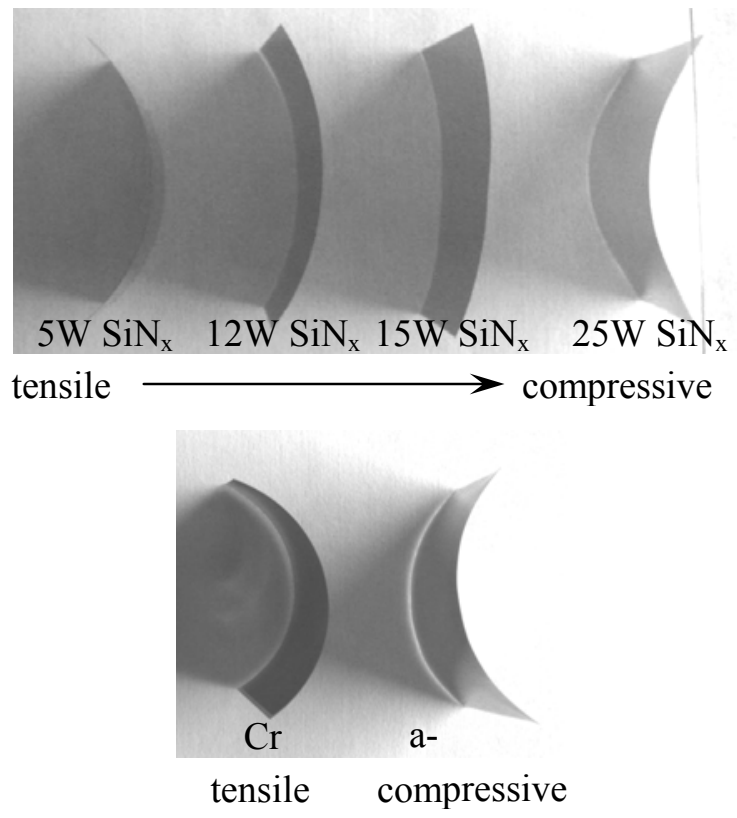

Figure 2.

Gleskova et al. 


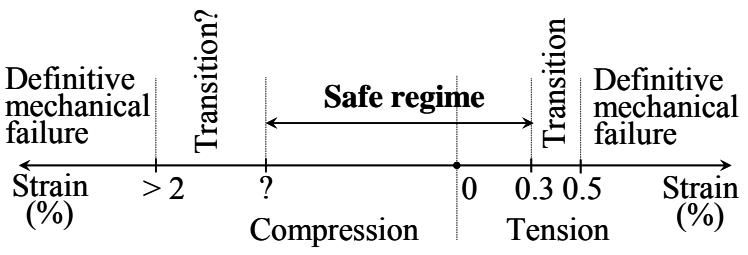

Figure 3.

Gleskova et al. 


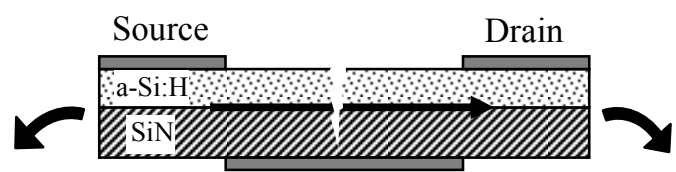

Bending

Gate

direction

Figure 4.

Gleskova et al. 


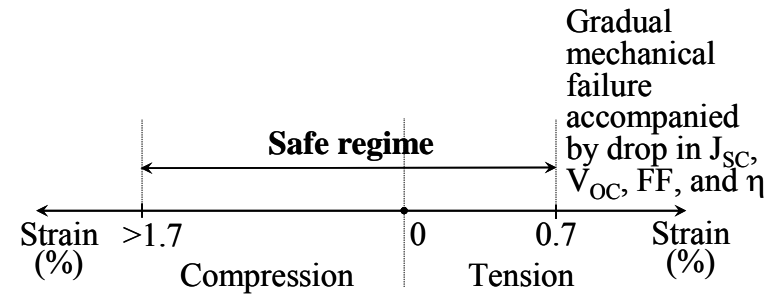

Figure 5.

Gleskova et al. 


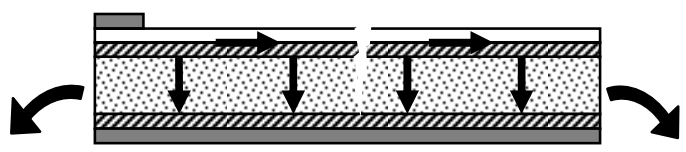

$\begin{array}{ll}\text { Bending } & \text { Layer description (from the top): } \\ \text { direction } & \text { Front contact metal } \\ & \text { Transparent conductor } \\ & \text { n-type layer } \\ & \text { Light absorbing layer } \\ \text { p-type layer } & \text { Back contact metal }\end{array}$

Figure 6.

Gleskova et al. 